\title{
Identification of the long non-coding RNA LET as a novel tumor suppressor in gastric cancer
}

\author{
JINGJING TIAN ${ }^{1}$, XIBAO HU $^{2}$, WEI GAO ${ }^{1}$, JIE ZHANG $^{1}$, MING CHEN $^{1}$, \\ XINRONG ZHANG ${ }^{1}$, JUNHONG MA ${ }^{1}$ and HONGXIA YUAN ${ }^{3}$ \\ ${ }^{1}$ Department of Gastroenterology, NanKai Hospital, Tianjin 300100; ${ }^{2}$ First Teaching Hospital and \\ ${ }^{3}$ School of Management, Tianjin University of Traditional Chinese Medicine, Tianjin 300193, P.R. China
}

Received November 23, 2015; Accepted November 15, 2016

DOI: $10.3892 / \mathrm{mmr} .2017 .6263$

\begin{abstract}
Long non-coding RNAs (lncRNAs) have emerged recently as important factors in regulating fundamental biological processes. Alterations in the expression and function of IncRNAs have been observed to promote tumor formation, progression and metastasis. Although downregulation of the expression levels of LET IncRNA in several tumors has been reported, its role in gastric cancer remains unknown. The aim of the present study was to investigate the expression and function of LET in gastric cancer development. The expression levels of LET in 37 pairs of gastric cancer and adjacent non-tumor tissues were detected by reverse transcription-quantitative polymerase chain reaction (RT-qPCR). In addition, LET expression in gastric cancer cell lines was analyzed by RT-qPCR assay analysis. Furthermore, the impact of LET on cell proliferation, migration and apoptosis were detected using the cell counting kit-8, wound scratch and ELISA assays, respectively. The results demonstrated that the expression level of LET was downregulated in gastric cancer tissues and cell lines (SGC-7901 and MGC-803) compared with normal tissues and a normal human gastric epithelial cell line (GES-1). Restoration of LET expression using a synthesized recombinant overexpression vector transfected into SGC-7901 and MGC-803 cells, significantly inhibited cell proliferation and migration, and promoted cell apoptosis in vitro. The present study is the first to demonstrate that LET may function as a tumor suppressor in gastric cancer. The results indicate that LET may be a promising biomarker and/or a therapeutic target for gastric cancer.
\end{abstract}

Correspondence to: Professor Hongxia Yuan, School of Management, Tianjin University of Traditional Chinese Medicine, 312 Anshan West Road, Nankai, Tianjin 300193, P.R. China E-mail: tjnkyhx@sina.com

Mr. Jingjing Tian, Department of Gastroenterology, NanKai Hospital, 122 Nankai Sanwei Road, Tianjin 300100, P.R. China E-mail: 25tianjingjing@sina.com

Key words: long non-coding RNAs, LET, gastric cancer, tumor suppressor

\section{Introduction}

Gastric cancer is the second leading cause of cancer-associated mortality worldwide, and is the most common gastrointestinal tumor in East Asia $(1,2)$. Gastrectomy remains the primary treatment for gastric cancer. Although the majority of patients at an early stage of gastric carcinoma may be cured by surgery, $>50 \%$ of patients do not survive due to carcinoma recurrence at an advanced stage of the disease, despite undergoing a curative gastrectomy (3). Therefore, an improved understanding of the pathogenesis and identification of the molecular alterations is essential for the development of diagnostic markers that may aid novel effective therapies for gastric cancer $(4,5)$.

Long non-coding RNA (lncRNAs), which are transcripts $>200$ bp in size with no protein-encoding function, represent a less investigated class of non-coding RNAs (6). Previous studies have reported that IncRNAs are crucial for the regulation of chromatin structure, gene expression and translational control (7-9). A significant number of studies have investigated small interfering RNAs and microRNAs, and their functions and molecular mechanisms have been illustrated in recent years $(10,11)$. However, our understanding of the function of lncRNAs in different diseases remains limited.

An increasing number of studies are investigating the role of lncRNAs in the origin and development of cancers, particularly digestive system cancers. High expression levels of PVT1 was demonstrated to be a potential candidate biomarker for gastric cancer, and promoted cell proliferation through the epigenetic regulation of p15 and p16 (12). In addition, upregulation of LINC00152 is reportedly correlated with depth of tumor invasion in patients with gastric cancer (13). The lncRNA CCAT2 is highly expressed and associated with poor prognosis in gastric cancer (14). Although several lncRNAs have been reported to function in the development of gastric cancer, the function of $<1 \%$ of the $30,000 \operatorname{lncRNAs}$ identified to date has been reported (15). Therefore, identification of gastric cancer-associated lncRNAs and investigation of their molecular mechanisms and biological functions, is essential for understanding the molecular biology of gastric cancer development.

A previous study demonstrated that LET was downregulated in gastric cancer and was associated with poor prognosis (16). The aim of the present study was to verify the expression of LET in gastric cancer tissues and assess 
its impact on gastric cancer cell proliferation, migration and apoptosis. The results demonstrated that LET may function as a tumor suppressor lncRNA in gastric cancer.

\section{Materials and methods}

Patients and specimens. A total of 37 cases of paired gastric cancer specimens used for the purposes of the present study, were collected from gastrectomy procedures at the Department of Gastroenterology, NanKai Hospital (Tianjin, China) between June 2008 and June 2014. Samples were collected from 17 male and 20 female patients. The average weight of male patients was $63.4 \pm 3.4 \mathrm{~kg}$ and the average weight (mean \pm standard deviation) of female patients was $52.6 \pm 3.8 \mathrm{~kg}$. The average age for male and female patients (mean \pm standard deviation) was $48.5 \pm 5.3$ years and $57.6 \pm 6.2$ years, respectively. All tissue samples were reviewed and diagnosed as gastric cancer according to the American Joint Committee on Cancer staging manual based on histopathological evaluation (17). None of the patients received percutaneous ablation, chemoembolization or radiotherapy prior to the gastrectomy operation. Written informed consent was provided by all patients, and the clinical research was approved by the Institutional Review Board of NanKai Hospital. All specimens were immediately frozen in liquid nitrogen for downstream analysis.

Cell culture and transfection. The human gastric cancer cell lines, SGC-7901 and MGC-803, were purchased from the American Type Culture Collection (Manassas, VA, USA). The normal human gastric epithelial cell line, GES-1, was obtained from the Cell Line Resource Center, Shanghai Institute of Biochemistry and Cell Biology, the Chinese Academy of Sciences. (Shanghai, China). Cells were maintained in Dulbecco's Modified Eagle's medium (Thermo Fisher Scientific, Inc., Waltham, MA, USA) containing 10\% fetal bovine serum at $37^{\circ} \mathrm{C}$ in a humidified atmosphere with $5 \% \mathrm{CO}_{2}$.

To investigate the function of LET in gastric cancer cells, an overexpression plasmid containing the LET lncRNA sequence was generated using gene recombination technology. The sequence of human LET was amplified by polymerase chain reaction (PCR) using the following primers: Forward, 5'-CGCGGATCCCTCACAGACAAA GGAGAGTCTGATG-3', and reverse, 5'-CCGGAATTCTG GGTGTTTTCATGTAGGAAATGGT-3'. All primers were obtained from Invitrogen (Invitrogen, CA, USA). PCR products were double-digested using BamHI and EcoRI restriction endonucleases (Takara Biotechnology Co., Ltd., Otsu, Japan) according to the manufacturer's instructions. The digested products were sub-cloned into the pcDNA3.1(+) vector (Invitrogen; Thermo Fisher Scientific, Inc.) to produce a pcDNA3.1(+)-LET fusion overexpression vector, and the sequence of pcDNA3.1(+)-LET were confirmed by Sanger DNA sequencing (Invitrogen; Thermo Fisher Scientific, Inc.). SGC-7901 and MGC-803 cells were transfected with $4 \mu \mathrm{g}$ pcDNA3.1(+)-LET recombinant overexpression vector or pcDNA3.1(+)empty vector using Lipofectamine 2000 (Invitrogen; Thermo Fisher Scientific, Inc.) at a ratio of 1.25:1.00 Lipofectamine:vector DNA.
Reverse transcription-quantitative PCR (RT-qPCR). Total RNA from gastric cancer tissues and cells $\left(1 \times 10^{6}\right)$ was extracted using TRIzol reagent (Invitrogen; Thermo Fisher Scientific, Inc.) according to the manufacturer's instructions. cDNA was synthesized from $1 \mu \mathrm{g}$ RNA using the PrimeScript RT kit (Takara Biotechnology Co., Ltd.). RT-qPCR reactions were performed using the $\mathrm{SYBR}^{\circledR}$ Premix EX Taq ${ }^{\mathrm{TM}}$ II PCR kit (Takara Biotechnology Co., Ltd.) according to the manufacturer's instructions, with the Roche LightCycler ${ }^{\circledR} 480$ Instrument II (Roche Diagnostics, Indianapolis, IN, USA). All primers were obtained from Invitrogen (Thermo Fisher Scientific, Inc.). The primer sequences were as follows: LET, forward, 5'-GGGAGTAAAGGGAAAGAGTTGC-3', and reverse, 5'-AGGCTGAGGAAGGTGGTATTGG-3'; GAPDH, forward, 5'-CATCAAGAAGGTGGTGAAGCAGG-3', and reverse, 5'-AAAGGTGGAGGAGTGGGTGTCG-3'. Data were collected and analyzed using Roche LightCycler ${ }^{\circledR} 480$ software (version 1.5; Roche Diagnostics). The expression of LET was normalized internally using the quantification cycle of the GAPDH housekeeping gene. The relative quantitative value was calculated using the $2^{-\Delta \Delta C a}$ method (18). Each experiment was performed in quintuplicate and repeated five times.

Cell counting kit-8 (CCK-8) assay. Cell proliferation was detected using the CCK- 8 assay kit (Dojindo Molecular Technologies, Inc., Kumamoto, Japan) according to the manufacturer's instructions. SGC-7901 and MGC-803 cells were seeded in 96-well plates at a density of $4 \times 10^{3}$ cells/well and cultured in normal medium for $24 \mathrm{~h}$, before they were transfected with the pcDNA3.1(+)-LET vector or the pcDNA3.1(+)empty vector. At $0,24,48$ and $72 \mathrm{~h}$ following transfection, the absorbance of each well at $450 \mathrm{~nm}$ (with $630 \mathrm{~nm}$ as the reference wavelength) was measured using an ELISA microplate reader (Bio-Rad Laboratories, Inc., Hercules, CA, USA). All assays were repeated at least three times.

Cell migration assay. The migratory ability of gastric cancer cells (SGC-7901 and MGC-803) was analyzed using an in vitro wound scratch assay. Cells $\left(\sim 5 \times 10^{7}\right)$ were cultured in a 6-well dish for $24 \mathrm{~h}$, before they were transfected with the pcDNA3.1(+)-LET vector or the pcDNA3.1(+)empty vector using Lipofectamine 2000. Vertical horizontal wounds were generated with a sterile $10 \mu \mathrm{l}$ pipette tip at $6 \mathrm{~h}$ following transfection. The cells were then washed with phosphate-buffered saline and maintained at $37^{\circ} \mathrm{C}$ in the incubator. The wound images were acquired with a digital camera system at 0 and $24 \mathrm{~h}$ after the wounds were generated. The width of wounds was measured using a standard caliper. All experiments were performed in triplicate and repeated at least three times.

Cell apoptosis assay. The extent of apoptosis was evaluated using a caspase 3 ELISA assay. SGC-7901 and MGC-803 gastric cancer cells were seeded in 6-well plates at a density of $2 \times 10^{6}$ cells/well and transfected with pcDNA3.1(+)-LET vector or pcDNA3.1(+)empty vector. At $48 \mathrm{~h}$ following transfection, cell apoptosis was determined by calculating the activity of caspase 3 using a caspase 3 ELISA kit (cat. no. KHO1091; Thermo Fisher Scientific, Inc.) and a Hoechst 33258 staining assay (Invitrogen; Thermo Fisher Scientific, Inc.) according to the manufacturer's instructions. The value of optical 
$\mathbf{A}$

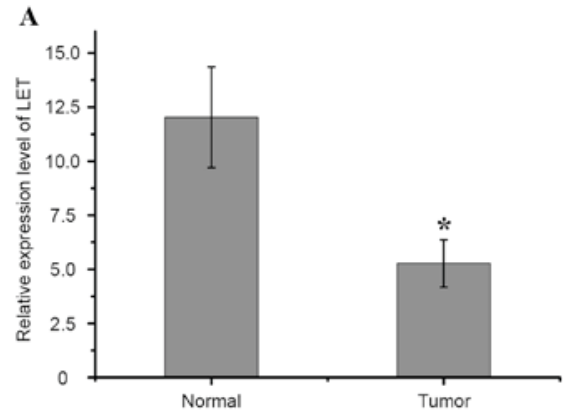

B

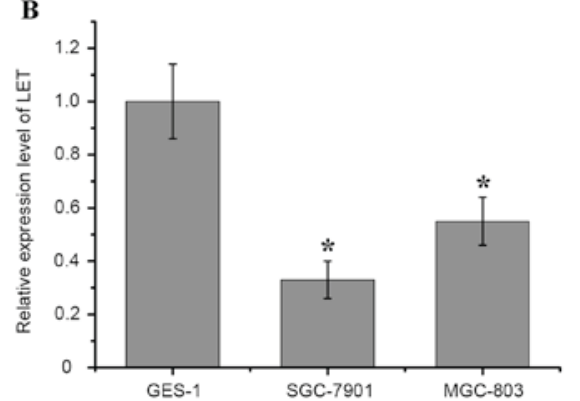

Figure 1. LET expression is downregulated in gastric cancer tissues and cell lines. (A) RT-qPCR analysis of LET expression in gastric cancer tissues compared with matched adjacent non-cancerous normal tissues $($ " $\mathrm{P}<0.01$ vs. Normal). (B) RT-qPCR analysis demonstrated that LET expression in SGC-7901 and MGC-803 gastric cancer cell lines was significantly reduced when compared with the normal human gastric epithelial mucosa cell line, GES-1 ("P $<0.05$ vs. GES-1). The $2^{-\triangle \Delta C q}$ method was used to quantify the expression levels of LET relative to GAPDH. Data are expressed as the mean \pm standard deviation $(\mathrm{n}=3)$. RT-qPCR, reverse transcription-quantitative polymerase chain reaction.

density at $405 \mathrm{~nm}$ was measured using an ELISA microplate reader (Bio-Rad Laboratories, Inc.). The ratio of cell apoptosis was calculated according to the formula: (ODxdilution factor $) /(10.5 x$ volume of sample in mlxreaction time in $\mathrm{min})$. The apoptosis of cell was also detected by a Zeiss LSM 710 laser scanning confocal microscopy (Carl Zeiss AG, Oberkochen, Germany) and analyzed using the Image-Pro Plus software program (version, 5.10; Media Cybernetics, Inc., Rockville, MD, USA). All experiments were performed in triplicate.

Statistical analysis. Statistical analysis of data was performed using SPSS software (version, 18.0; SPSS, Inc., Chicago, IL, USA). The paired $t$-test was used to compare LET expression in gastric cancer tissues with matched non-tumor tissues. The independent samples $t$-test was used to analyze the remaining data. $\mathrm{P}<0.05$ was considered to indicate a statistically significant difference.

\section{Results}

LET expression is downregulated in gastric cancer tissues and cell lines. A previous study reported that LET is downregulated in gastric cancer tissues (17). To assess the function of LET in gastric cancer in the present study, the expression level of LET in 37 gastric cancer tissues and matched adjacent non-tumor tissues was examined by RT-qPCR analysis. As showed in Fig. 1A, the expression of LET in gastric cancer tissues was significantly lower when compared with matched adjacent
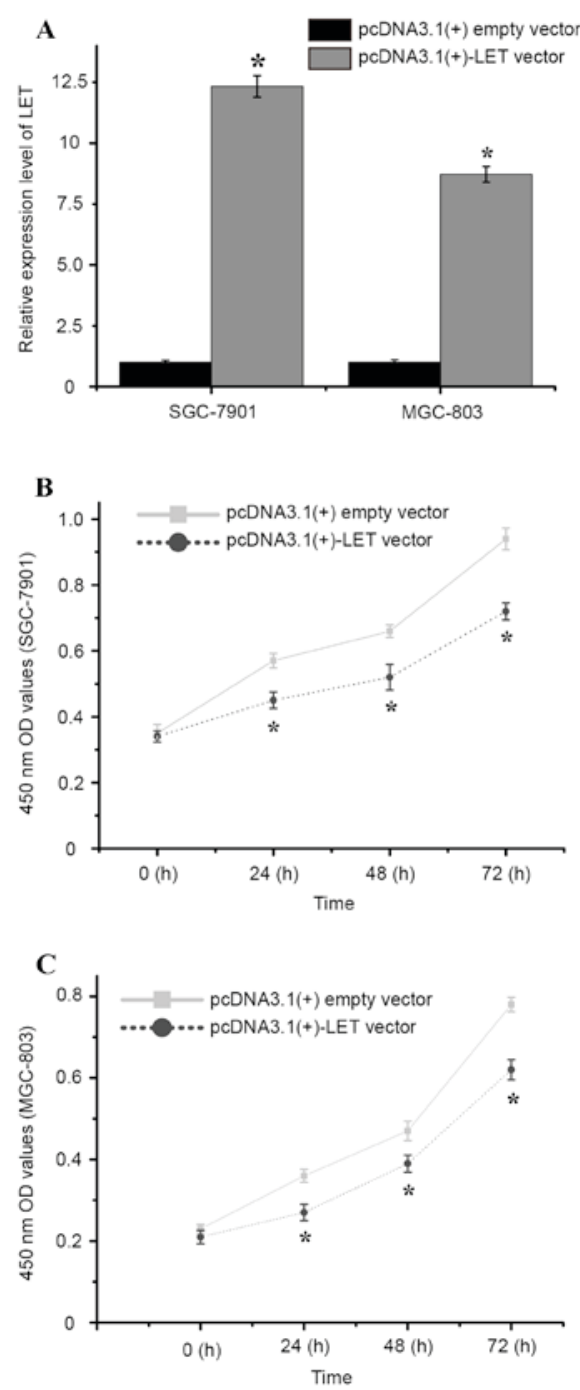

Figure 2. Restoration of LET expression suppresses gastric cancer cell proliferation in vitro. (A) Reverse transcription-quantitative polymerase chain reaction analysis of LET expression following transfection of SGC-7901 and MGC-803 cells with pcDNA3.1(+)-LET or pcDNA3.1(+) empty vectors $\left[{ }^{*} \mathrm{P}<0.01\right.$ vs. pcDNA3.1(+)empty vector]. The cell counting kit-8 assay demonstrated a significant reduction in cell proliferation in pcDNA3.1(+)-LET vector-transfected (B) SGC-7901 and (C) MGC-803 cells when compared with the pcDNA3.1(+)empty vector-transfected cells $\left[{ }^{*} \mathrm{P}<0.05\right.$ vs. pcDNA3.1(+)empty vector]. Data are expressed as the mean \pm standard deviation. Experiments were performed in duplicate and repeated three times. OD, optical density.

non-tumor tissues $(\mathrm{P}<0.01)$. LET expression in gastric cancer cell lines and the human gastric epithelial mucosa cell line GES-1 was then assessed by RT-qPCR analysis. Consistent with the results shown in Fig. 1A, LET expression was significantly downregulated in SGC-7901 $(\mathrm{P}=0.017)$ and MGC-803 $(\mathrm{P}=0.032)$ cells compared with GES-1 cells (Fig. 1B). These results suggest that LET expression is downregulated in gastric cancer tissues and cell lines.

Restoration of LET expression suppressed gastric cancer cell proliferation in vitro. To investigate the functional role of LET in gastric cancer cell proliferation, the pcDNA3.1(+)-LET vector or pcDNA3.1(+)empty vector was transfected into SGC-7901 and MGC-803 cells, and cell proliferation was examined using a CCK-8 assay. As shown in Fig. 2A, SGC-7901 
$\mathbf{A}$

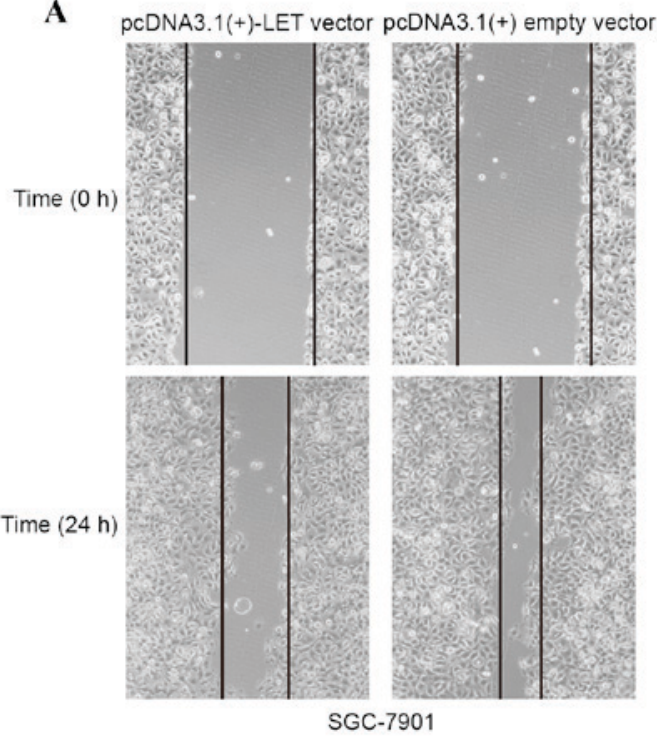

B pCDNA3.1(+)-LET vector pcDNA3.1(+) empty vector

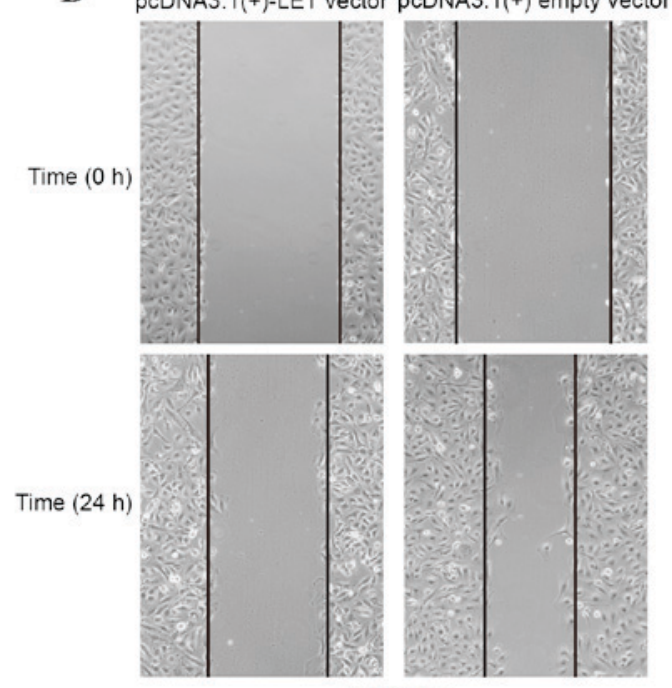

Figure 3. Restoration of LET expression inhibits gastric cancer cell migration in vitro. A wound healing assay was performed to determine the proliferation of pcDNA3.1(+)-LET vector and pcDNA3.1(+)empty vector-transfected (A) SGC-7901 and (B) MGC-803 cells at 24 h following transfection. Data are presented as the mean \pm standard deviation. Each experiment was repeated three times independently.
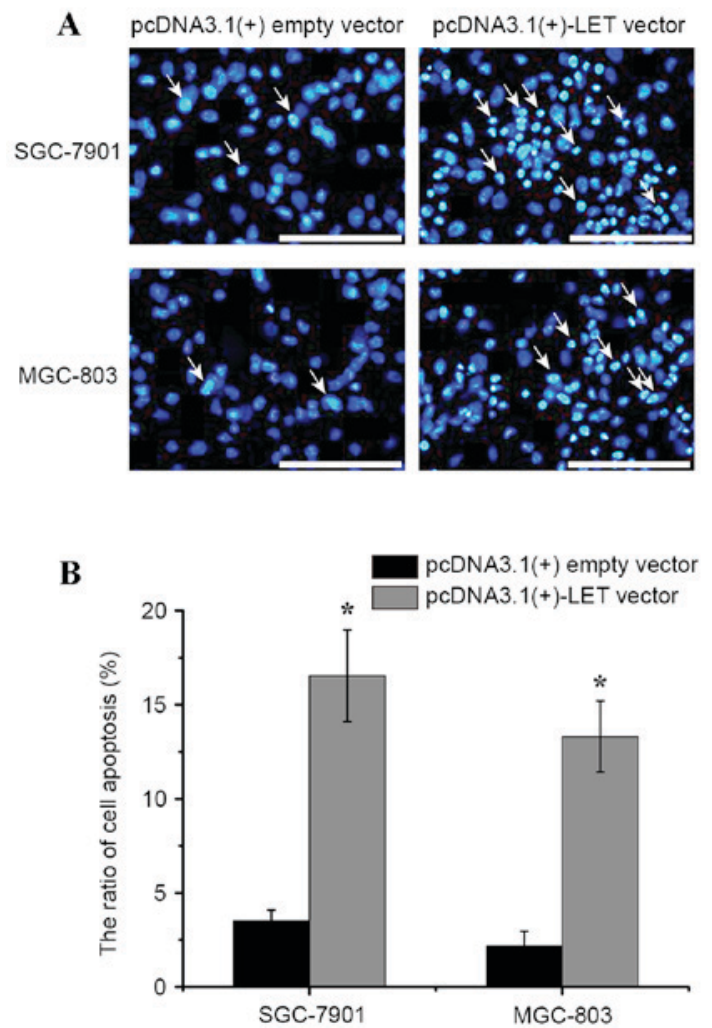

Figure 4. Restoration of LET expression induces gastric cancer cell apoptosis in vitro. (A) Laser scanning confocal microscopy images of Hoechst 33258-stained SGC-7901 and MGC-803 cells transfected with the pcDNA3.1(+)-LET vector or the pcDNA3.1(+)empty vector (scale bar, $20 \mu \mathrm{m}$ ). White arrows indicate apoptotic cells. (B) Comparison of SGC-7901 and MGC-803 cell apoptosis rates following transfection with pcDNA3.1(+)-LET vector or pcDNA3.1(+)empty vector. Data are presented as the mean \pm standard deviation. ${ }^{*} \mathrm{P}<0.01$ vs. pcDNA3.1(+)empty vector.

and MGC-803 cells transfected with the pcDNA3.1(+)-LET vector demonstrated a significant increase in LET expression when compared with pcDNA3.1+ empty vector-transfected cells $(\mathrm{P}<0.01$; Fig. 2A). The CCK-8 assay was then performed and the optical density values of the pcDNA3.1(+)-LET vector or pcDNA3.1(+)empty vector-transfected cells were measured at $0,24,48$ and $72 \mathrm{~h}$ following transfection. The results demonstrated that the relative cell proliferation of pcDNA3.1(+)-LET vector-transfected SGC-7901 cells was significantly decreased at 24,48 and $72 \mathrm{~h}$ when compared with pcDNA3.1(+)-transfected cells $(5.43,6.07$ and $9.21 \%$, respectively; P<0.01; Fig. 2B). Similarly, the relative cell proliferation of pcDNA3.1(+)-LET vector-transfected MGC-803 cells was significantly decreased at 24,48 and $72 \mathrm{~h}$ following transfection when compared with pcDNA3.1(+)-transfected cells (4.09, 3.88 and $7.47 \%$, respectively; $\mathrm{P}<0.01$; Fig. 2 C). These results indicate that overexpression LET may significantly inhibit cell the proliferation of gastric cancer cells in vitro.

Restoration of LET expression inhibits gastric cancer cell migration in vitro. The effect of LET overexpression on gastric cancer cell migration was determined using a wound healing assay. As shown in Fig. 3A and B, the wound widths of SGC-7901 and MGC-803 cells transfected with the pcDNA3.1(+)-LET vector were markedly greater when compared with the pcDNA3.1(+)empty vector-transfected cells. The results demonstrate that restoration LET expression may inhibit gastric cancer cell migration.

Restoration of LET expression induces gastric cancer cell apoptosis in vitro. LncRNAs have been reported to serve a crucial role in cell apoptosis, particularly in mediating escape from apoptosis in cancer cells (19). To determine the effect of LET on gastric cancer cell apoptosis, a caspase 3 ELISA assay was used to measure the rate of apoptosis. As revealed in Fig. 4, the apoptosis rate of SGC-7901 cells transfected with pcDNA3.1(+)-LET vector was significantly higher when compared with the pcDNA3.1(+)empty vector-transfected cells $(16.53 \%$ vs. $3.35 \%$; $\mathrm{P}<0.01)$. Similarly, the apoptosis rate 
of pcDNA3.1(+)-LET vector-transfected MGC-803 cells was significantly higher when compared with pcDNA3.1(+)empty vector-transfected cells (13.30\% vs. $2.19 \%$; $\mathrm{P}<0.01$; Fig. 4B). The results demonstrate that overexpression of LET may promote apoptosis of gastric cancer cells. The results presented thus far, identify LET IncRNA as a novel tumor suppressor in gastric cancer.

\section{Discussion}

Gastric cancer is one of the most common types of digestive tumors (20). It is the second most frequent cause of cancer-associated mortality worldwide and presents a major public health issue (21). Despite significant advances in cancer therapy, major limitations in the management of gastric cancer remain. A large number of patients are diagnosed with advanced gastric cancer and have a poor prognosis.

Emerging evidence suggests that lncRNAs serve essential roles in the modulation of tumor behavior through various complex mechanisms, such as modulating gene transcription and epigenetic signaling pathways $(22,23)$. A recent study suggested that the HOX transcript antisense RNA lncRNA may promote the malignant growth of human liver cancer stem cells through downregulation of SET domain containing 2 (24). In addition, the intronic prostate cancer antigen 3 lncRNA regulates the prune homolog 2 suppressor in prostate cancer (25). Furthermore, increased HOXA transcript at the distal tip lncRNA expression is correlated with prognosis and progression in tongue squamous cell carcinoma (26). Silencing prostate cancer associated transcript-1 induces cell growth arrest and apoptosis in human bladder cancer (27). However, limited data are available regarding the expression and function of lncRNAs in gastric cancer.

The LET lncRNA gene is located on chromosome 15q24.1. LET exhibits differential expression patterns in various tumors (16,28-30). Previous studies have demonstrated that LET expression is downregulated in cervical and gastric cancer $(16,28)$. However, to the best of our knowledge, the precise function of LET has not yet been reported in gastric cancer. In the present study, the expression of LET in gastric cancer tissues and cell lines was first examined. Consistent with previously reported results, the expression of LET was downregulated in gastric cancer tissues when compared with matched adjacent normal tissues. In addition, LET expression was decreased in two gastric cancer cell lines (SGC-7901 and MGC-803) when compared with a human gastric epithelial mucosa cell line (GES-1). These results provided a strong rationale for subsequent functional experiments.

To further understand the biological functions of LET in gastric cancer cells, cell proliferation, migration and apoptosis was examined by applying a gain-of-function approach. Transfection of SGC-7901 and MGC-803 cells with the pcDNA3.1(+)-LET vector led to a significant reduction in cell proliferation and migration, and increased apoptosis when compared with pcDNA3.1(+)empty vector-transfected cells. These results suggest that LET may function as a tumor suppressor gene in the occurrence and development of gastric cancer.

In conclusion, the results of the present study confirm that LET is significantly downregulated in human gastric cancer tissues and cells. In addition, this study is the first to demonstrate that LET serves a tumor suppressive role in gastric cancer by influencing cellular migration, proliferation and apoptosis. Therefore, LET presents a promising biomarker and/or a therapeutic target for gastric cancer.

\section{References}

1. Siegel R, Naishadham D and Jemal A: Cancer statistics, 2013. CA Cancer J Clin 63: 11-30, 2013.

2. Hu N, Wang Z, Song X, Wei L, Kim BS, Freedman ND, Baek J, Burdette L, Chang J, Chung C, et al: Genome-wide association study of gastric adenocarcinoma in Asia: A comparison of associations between cardia and non-cardia tumours. Gut 65 : 1611-1688, 2016.

3. Nakajima T: Gastric cancer treatment guidelines in Japan. Gastric Cancer 5: 1-5, 2002.

4. Vogiatzi P, Vindigni C, Roviello F, Renieri A and Giordano A: Deciphering the underlying genetic and epigenetic events leading to gastric carcinogenesis. J Cell Physiol 211: 287-295, 2007.

5. Crew KD and Neugut AI: Epidemiology of gastric cancer. World J Gastroenterol 12: 354-362, 2006.

6. Costa FF: Non-coding RNAs: Meet thy masters. Bioessays 32: 599-608, 2010.

7. Liu J, Shen L, Yao J, Li Y, Wang Y, Chen H and Geng P: Forkhead box $\mathrm{C} 1$ promoter upstream transcript, a novel long non-coding RNA, regulates proliferation and migration in basal-like breast cancer. Mol Med Rep 11: 3155-3159, 2015.

8. Tian F, Tang Z, Song G, Pan Y, He B, Bao Q and Wang S: Loss of imprinting of IGF2 correlates with hypomethylation of the H19 differentially methylated region in the tumor tissue of colorectal cancer patients. Mol Med Rep 5: 1536-1540, 2012.

9. Eades G, Wolfson B, Zhang Y, Li Q, Yao Y and Zhou Q: lincRNA-RoR and miR-145 regulate invasion in triple-negative breast cancer via targeting ARF6. Mol Cancer Res 13: 330-338, 2015.

10. Rusek AM, Abba M, Eljaszewicz A, Moniuszko M, Niklinski J and Allgayer H: MicroRNA modulators of epigenetic regulation, the tumor microenvironment and the immune system in lung cancer. Mol Cancer 14: 34, 2015.

11. Cheng CJ, Bahal R, Babar IA, Pincus Z, Barrera F, Liu C, Svoronos A, Braddock DT, Glazer PM, Engelman DM, et al: MicroRNA silencing for cancer therapy targeted to the tumour microenvironment. Nature 518: 107-110, 2015.

12. Kong R, Zhang EB, Yin DD, You LH, Xu TP, Chen WM, Xia R, Wan L, Sun M, Wang ZX, et al: Long noncoding RNA PVT1 indicates a poor prognosis of gastric cancer and promotes cell proliferation through epigenetically regulating $\mathrm{p} 15$ and $\mathrm{p} 16$. Mol Cancer 14: 82, 2015.

13. Pang Q, Ge J, Shao Y, Sun W, Song H, Xia T, Xiao B and Guo J: Increased expression of long intergenic non-coding RNA LINC00152 in gastric cancer and its clinical significance. Tumour Biol 35: 5441-5447, 2014.

14. Wang CY, Hua L, Yao KH, Chen JT, Zhang JJ and Hu JH: Long non-coding RNA CCAT2 is up-regulated in gastric cancer and associated with poor prognosis. Int J Clin Exp Pathol 8: 779-785, 2015.

15. Gan L, Xu M, Zhang Y, Zhang X and Guo W: Focusing on long noncoding RNA dysregulation in gastric cancer. Tumour Biol 36: 129-141, 2015.

16. Zhou B, Jing XY, Wu JQ, Xi HF and Lu GJ: Down-regulation of long non-coding RNA LET is associated with poor prognosis in gastric cancer. Int J Clin Exp Pathol 7: 8893-8898, 2014.

17. Strong VE, D'Amico TA, Kleinberg L and Ajani J: Impact of the 7th Edition AJCC staging classification on the NCCN clinical practice guidelines in oncology for gastric and esophageal cancers. J Natl Compr Canc Netw 11: 60-66, 2013.

18. Schmittgen TD and Livak KJ: Analyzing real-time PCR data by the comparative C(T) method. Nat Protoc 3: 1101-1108, 2008.

19. Li J, Tian H, Yang J and Gong Z: Long Noncoding RNAs regulate cell growth, proliferation, and apoptosis. DNA Cell Biol 35: 459-470, 2016.

20. Yamamoto H, Watanabe Y, Maehata T, Morita R, Yoshida Y, Oikawa R, Ishigooka S, Ozawa S, Matsuo Y, Hosoya K, et al: An updated review of gastric cancer in the next-generation sequencing era: Insights from bench to bedside and vice versa. World J Gastroenterol 20: 3927-3937, 2014. 
21. Smith MG, Hold GL, Tahara E and El-Omar EM: Cellular and molecular aspects of gastric cancer. World J Gastroenterol 12: 2979-2990, 2006.

22. Szczesniak MW and Makalowska I: lncRNA-RNA interactions across the human transcriptome. PLoS One 11: e0150353, 2016.

23. Liu B, Sun L and Song E: Pinched by RNA 'fingers': Long noncoding RNAs hitting signal transduction pathways. Mol Cell Oncol 3: e1046582, 2016.

24. Li H, An J, Wu M, Zheng Q, Gui X, Li T, Pu H and Lu D: LncRNA HOTAIR promotes human liver cancer stem cell malignant growth through downregulation of SETD2. Oncotarget 6 : 27847-27864, 2015.

25. Salameh A, Lee AK, Cardó-Vila M, Nunes DN, Efstathiou E, Staquicini FI, Dobroff AS, Marchiò S, Navone NM, Hosoya $\mathrm{H}$, et al: PRUNE2 is a human prostate cancer suppressor regulated by the intronic long noncoding RNA PCA3. Proc Natl Acad Sci USA 112: 8403-8408, 2015

26. Zhang H, Zhao L, Wang YX, Xi M, Liu SL and Luo LL: Long non-coding RNA HOTTIP is correlated with progression and prognosis in tongue squamous cell carcinoma. Tumour Biol 36: 8805-8809, 2015.
27. Liu L, Liu Y, Zhuang C, Xu W, Fu X, Lv Z, Wu H, Mou L, Zhao G, Cai Z and Huang W: Inducing cell growth arrest and apoptosis by silencing long non-coding RNA PCAT-1 in human bladder cancer. Tumour Biol 36: 7685-7689, 2015.

28. Jiang S, Wang HL and Yang J: Low expression of long non-coding RNA LET inhibits carcinogenesis of cervical cancer. Int J Clin Exp Pathol 8: 806-811, 2015.

29. Ma MZ, Kong X, Weng MZ, Zhang MD, Qin YY, Gong W, Zhang WJ and Quan ZW: Long non-coding RNA-LET is a positive prognostic factor and exhibits tumor-suppressive activity in gallbladder cancer. Mol Carcinog 54: 1397-1406, 2015.

30. Yang F, Huo XS, Yuan SX, Zhang L, Zhou WP, Wang F and Sun SH: Repression of the long noncoding RNA-LET by histone deacetylase 3 contributes to hypoxia-mediated metastasis. Mol Cell 49: 1083-1096, 2013 